\title{
ELASTOHYDRODINAMIC ANALYSIS OF THE CONROD SMALL-END OF A HIGH PERFORMANCE MOTORBIKE ENGINE VIA A MASS CONSERVING CAVITATION ALGORITHM
}

\author{
Luca Nicolò Mastrandrea \\ Engineering Department Enzo Ferrari, University \\ of Modena and Reggio Emilia \\ Modena, Italy \\ Daniele Dini \\ Mechanical Engineering, Imperial College \\ London, United Kingdom
}

\author{
Matteo Giacopini \\ Engineering Department Enzo Ferrari, University \\ of Modena and Reggio Emilia \\ Modena, Italy \\ Enrico Bertocchi \\ Engineering Department Enzo Ferrari, University \\ of Modena and Reggio Emilia \\ Modena, Italy
}

\begin{abstract}
In this contribution a complementarity formulation for the solution of EHL problem in presence of cavitation is employed in order to investigate the tribological behavior of the conrod small-end of a high performance motorbike engine. The influence of different physical and geometrical parameters is discussed. In particular, the clearance between the conrod smallend and the piston pin, the lubricant physical properties, the surface roughness and the stiffness of the piston pin are investigated, thus providing preliminary guidelines for the correct design of the coupling. Due to the negligible influence of the transversal forces acting on the conrod small-end and of the relative sliding speed between the mating surfaces, a two symmetrical model of the assembly is prepared and results are compared with those obtained adopting a simply symmetrical model.
\end{abstract}

\section{INTRODUCTION}

During the last decades, the internal combustion engine performance has constantly increased. At the same time, there has been a growing emphasis on the analysis of the aspects related to the efficiency, to the reliability and to the cost of manufacturing of modern engines. To satisfy the above targets, a constant improvement and optimization of the design process of engines components is therefore necessary.

In particular, engine bearings represent one of the main responsible of friction losses and their lubrication performance has a crucial influence on the operating condition of an engine.
Consequently, to develop numerical methodology for the analysis of the lubrication behavior of these bearings can be considered an imperative concern for design engineers.

In particular, the conrod small-end bearing is one of the most critical engine parts from a tribological point of view. Moreover, considering the recent trend of removing the conrod small-end bush with the aim of avoiding problems related to the coupling between the bush and the conrod [1], and to save money by removing bush press-fit and machining, the correct prediction of the tribological behavior of the conrod small-end/piston pin interface has become fundamental. Fig.1 provides a schematic drawing of a typical conrod small-end assembly.

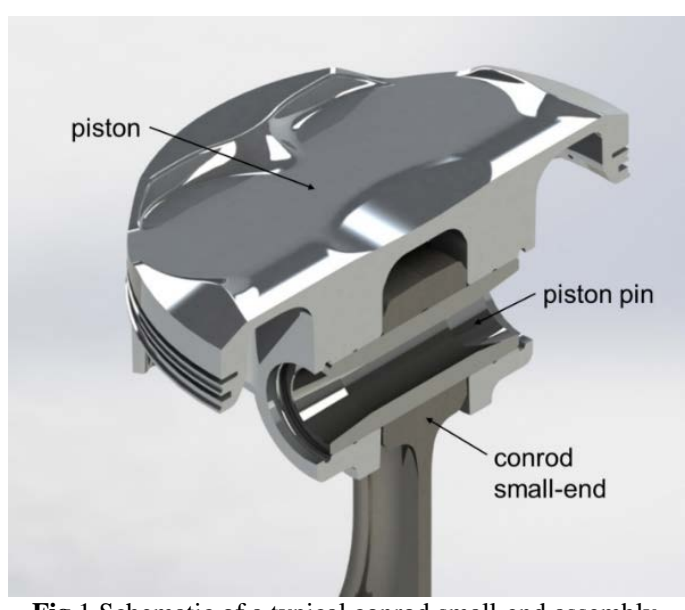

Fig.1 Schematic of a typical conrod small-end assembly. 
Differently from the conrod big-end, that has been widely investigated both theoretically and experimentally in several contributions in the pertinent literature [2, 3, 4 and 5], only few contributions can be found focusing on the tribological behavior of the conrod small-end [6, 7, 8, 9 and 10]. This lack of modeling is probably related to the difficulties that have to be faced in the determination of the operating conditions of the coupling between the conrod small-end and the piston pin, in terms of relative speed between the contact surfaces and of the lubricant feeding mechanism. In fact, the way the hydrodynamic lubrication process sustains the external load in the conrod bigend bearing strongly differs from the one encountered in the small-end counterpart [10]. In the conrod big-end, the hydrodynamic load capacity is mainly generated by the high relative rotational speed between the conrod and the crankshaft. On the contrary, the relative rotational speed between the conrod small-end and the piston pin is low and only a partial oscillation between the mating surfaces occurs. Moreover, the piston pin generally floats with respect to the two other components in contact with it, namely the conrod and the piston. For this reason, it is not possible to exactly define the relative speed between the mating surfaces of the small-end and of the piston pin. Therefore, the dominant effect in the lubrication of the small-end is the film squeeze caused by the alternating combustion/inertial loads, acting on the coupling, while sliding hydrodynamic effects are usually negligible.

In particular, the radial motion of the piston pin in the small-end bearing is characterized by both positive and negative squeeze. While the first one is well known and it corresponds to the case of a normal approach of two solid elements separated by a fluid film (thus providing the capacity of sustaining external loads by increasing the lubricant film pressure), the second is not as well understood. In fact, a normal separation of two surfaces fully submerged in a liquid produces a sudden and rapid decrease of the film pressure, which may induce fluid cavitation onset and development [11]. Therefore, the correct cavitation modeling and prediction is a fundamental aspect that has to be taken into account.

In the present contribution, the algorithm proposed by Giacopini et. al. [12, 13], based on a mass-conserving formulation of the Reynolds equation suitably recasted in terms of the two complementarity variables, namely the hydrodynamic pressure and the void-fraction, has been employed for the analysis of the tribological behavior of the conrod small-end of a high performance motorbike engine.

The correct prediction of the cavitation and the location of the boundaries between cavitated and active areas is guaranteed by the complementarity nature of the two chosen variables and the approach naturally leads to mass conservation and unconditional convergence. The EHL problem is numerically solved using a formulation based on the Finite Element Method [13].

The influence of several parameters governing the oil feeding process, such as the clearance between the piston pin and the conrod small-end, the lubricant properties, the roughness of the mating surfaces and specific design configurations like piston pin stiffness, has been investigated. The variation of oil film thickness, pressure distribution, load-carrying capacity and locus of pin centre have been evaluated.

\section{NOMENCLATURE}

$R_{i} \quad$ small-end inner radius

$T$ small-end axial thickness

$U$ sliding velocity

c small-end radial clearance

$h$ film thickness

$h_{\mathrm{d}}$ elastic deflection induced by dry contact pressure

$h_{\mathrm{e}}$ elastic deflection induced by hydrodynamic pressure

$h_{\mathrm{g}}$ geometric thickness

$h_{0}$ film thickness at which dry contact occurs

$C$ Compliance matrix

$p$ hydrodynamic pressure

$p_{d}$ dry contact pressure

$p_{\text {tot }}$ total pressure

$p_{c}$ cavitation pressure

$r \quad$ void fraction

$\mu$ dynamic viscosity

$\rho$ density

$\rho_{0}$ density at a given pressure

$\rho_{c}$ density at cavitation pressure

$\omega$ angular velocity

$e \quad$ journal centre position

\section{COMPLEMENTARITY FORMULATION OF THE REYNOLDS EQUATION}

In this section the complementarity formulation of the Reynolds equation is presented. Consider the Reynolds equation in its one dimensional form for unsteady and compressible flow:

$$
\frac{\partial}{\partial x}\left[\frac{\rho h^{3}}{6 \mu} \frac{\partial p}{\partial x}\right]-2 \frac{\partial}{\partial t}[\rho h]-U \frac{\partial}{\partial x}[\rho h]=0
$$

where $h$ is the film thickness, $U$ the sliding speed, $\mu$ the fluid viscosity and $\rho$ the density of the lubricant.

In the definition of a complementarity formulation, two aspects must be considered: (i) the identification of the complementarity variables and (ii) the definition of the functional connection that relates these variables. The complementarity variables adopted in the formulation employed in this paper are the same proposed in [13], namely the hydrodynamic pressure, $p$, and the void fraction, $r$, defined as:

$$
r=1-\frac{\rho}{\rho_{0}}
$$

where $\rho$ is the density of the mixture of oil and gases in the cavitated area and $\rho_{0}$ the density of the lubricant at the given pressure.

Eq.1 is valid both in the full film region (active region) and in the cavitated (non-active) region. In the active region the lubricant is fully composed of liquid, so $\rho$ equals $\rho_{0}$, while in the non-active region the fluid cavitates, becoming a mixture of liquid and vapour characterized by a density, $\rho=(x, t, p)$, which is always lower than or equal to $\rho_{0}$. It follows that $r \geq 0$ in the 
whole domain. In fact, in the active region $\rho$ equals $\rho_{0}$ and, therefore, $r=0$, while, in the cavitated region, $\rho$ is lower than $\rho_{0}$, thus, $r$ is greater than zero.

On the other side, the pressure, $p$, is greater than zero in the active region and it could be considered equal to zero in the cavitated counterpart, i.e. $p_{\mathrm{c}}=0$. It follows that $p \geq 0$ in the whole domain (note that if $p_{\mathrm{c}} \neq 0$, the sign restriction applies to the difference between the fluid pressure and the vapour pressure: $p-p_{c} \geq 0$ ). Therefore, the product $(p \cdot r)$ is null in the whole domain and the two variables $p$ and $r$ are complementary. With few simple manipulations, it is possible to write Eq.1 in terms of the two complementarity variables defined:

$$
\begin{aligned}
\frac{\partial}{\partial x}\left[\frac{h^{3}}{6 \mu} \frac{\partial p}{\partial x}\right]-\frac{\partial}{\partial x}[ & {\left[r \frac{h^{3}}{6 \mu} \frac{\partial p}{\partial x}\right]-2 \frac{\partial}{\partial t}[h]+2 \frac{\partial}{\partial t}[r h] } \\
& -U \frac{\partial}{\partial x}[h]+U \frac{\partial}{\partial x}[r h]=0
\end{aligned}
$$

Due to the orthogonal property of some terms of Eq.3, it can be simplified and the complementarity formulation based on the Reynolds equation and related to the problem of cavitation in lubricant films can be defined as follows: find $p$ and $r$ such that

$$
\begin{aligned}
\frac{\partial}{\partial x}\left[\frac{h^{3}}{6 \mu} \frac{\partial p}{\partial x}\right]-2 \frac{\partial}{\partial t}[h]+ & 2 \frac{\partial}{\partial t}[r h]-U \frac{\partial}{\partial x}[h]+U \frac{\partial}{\partial x}[r h]=0 \\
p & \geq 0 \\
r & \geq 0 \\
p \cdot r & =0
\end{aligned}
$$

\section{ELASTIC DEFLECTION}

In real journal bearings analysis, the elastic deformation of the components is not negligible as it strongly affect the results in terms of pressure, void fraction and, especially, journal path. Therefore, the HL complementarity formulation of the Reynolds equation in the presence of cavitation presented in Eq.4 has been improved to handle the elastic deflections of the contacting bodies. The EHL complementarity problem can be written as:

$$
\begin{gathered}
\frac{\partial}{\partial x}\left[\frac{h(p)^{3}}{6 \mu} \frac{\partial p}{\partial x}\right]-2 \frac{\partial}{\partial t}[h(p)]+2 \frac{\partial}{\partial t}[r h(p)]-U \frac{\partial}{\partial x}[h(p)] \\
+U \frac{\partial}{\partial x}[r h(p)]=0 \\
h(p)=h_{\mathrm{g}}+h_{\mathrm{e}}=h_{\mathrm{g}}+l p \\
p \geq 0 \\
r \geq 0 \\
p \cdot r=0
\end{gathered}
$$

where the total film thickness $h(p)$ is given by the sum of the geometric thickness $h_{\mathrm{g}}$ and the elastic deflection $h_{\mathrm{e}}$, and $l$ is the linear integral operator that gives the elastic deflection of the solid surfaces $h_{\mathrm{e}}$ as a function of the pressure $p$. In a discretised domain, it is possible to evaluate the elastic deflection at every point of the domain by an interpolation of the nodal values once proper shape functions have been introduced.

The values of the nodal displacements can be obtained by multiplying a matrix carrying information about the compliance of the domain, $C$, with the nodal pressures vector, $p$ (i.e. in a discretised geometry the operator $l$ is represented by a compliance matrix $C$ ).

The non-linear nature of the coupling of the Reynolds equation and the elastic deflection requires the implementation of an iterative procedure. At each step $i, h$ is evaluated with the pressure distribution $p_{i-1}$, computed at the previous step. Then the Reynolds equation in terms of $p$ and $r$ is solved giving the new pressure distribution $p_{i}$. This procedure loops until suitable convergence criteria are satisfied. However, such explicit method is not appropriate for the highly non-linear behaviour of the system of Eq.5, which describes the EHL problem.

Convergence issues arise and proper under-relaxation factors are required. Nevertheless, the introduction of under-relaxation techniques increases the computational cost and the time required to obtain the numerical solution. Moreover, the convergence is far from being guaranteed. Therefore, a suitable form of the Newton method has been introduced to tackle this problem. Following the formulation developed by Ruskell [14], the system of Eq.5 can be written as a non-linear integrodifferential equation for $p$ :

$$
F(p)=0
$$

Then, it is possible to use the Newton's method to obtain at each step a better distribution $p_{i}$ :

$$
F\left(p_{i}\right)+F^{\prime}\left(p_{i-1}\right) \varepsilon_{i-1}=0 .
$$

where $F^{\prime}$ is the functional derivative of $F$ and $\varepsilon_{i-1}$ is the error $\varepsilon_{i-}$ ${ }_{1}=p_{i}-p_{i-1}$. The functional derivative or Frèchet derivative of $F$ is defined by:

$$
F^{\prime}(p) \varepsilon=\left.\frac{d}{d \delta} F(p+\delta \varepsilon)\right|_{\delta=0} .
$$

This approach differs from more conventional methods as it incorporates both the hydrodynamic and the elastic equations in a single expression. In this way, the stability problems that arise during the iterative process when solving the system of Eq.5 separately are overcome. A clear and more detailed discussion on the advantages of this approach is presented in [14].

In order to numerically solve the problem, the system of Eq.5 is discretized via Finite Element technique. For further details refer to [15].

\section{MIXED LUBRICATION}

When the load capacity of the fluid film is not able to sustain the external loads, a direct contact between the solid surfaces asperities occurs. In this case, the supporting load is provided partly by the hydrodynamic oil film pressure and partly by the asperities dry contact pressure. This lubrication regime is well known as the mixed lubrication regime.

Therefore, the proposed algorithm has been further improved including the possibility to handle a direct contact between the surfaces asperities. In particular, following [16], the mixed lubrication problem has been described in terms of a complementarity problem, being the two complementarity variables the dry contact pressure, $p_{\mathrm{d}}$, and the gap between the 
surfaces asperities, $h$ - $h_{0}$, where $h_{0}$ is the film thickness at which dry contact occurs (usually related to the surface roughness)and $h$ is the total thickness defined as:

$$
h=h_{\mathrm{g}}+h_{\mathrm{e}}+h_{\mathrm{d}}=h_{\mathrm{g}}+h_{\mathrm{e}}+\mathrm{C} p_{\mathrm{d}}
$$

where $C$ is the compliance matrix of the assembly, $h_{\mathrm{g}}$ is the film thickness due to the geometrical relative position of the pin and of the small-end, $h_{\mathrm{e}}$ is the film thickness due to the elastic deflection of the mating surfaces induced by the hydrodynamic pressure.

In fact, where the gap $h-h_{0}$ is greater than zero no contact occurs, thus $p_{\mathrm{d}}$ is equal to zero. On the other hand, when the gap $h-h_{0}$ is equal to zero, the contact pressure, $p_{\mathrm{d}}$, becomes greater than zero.

In summary, the linear complementarity problem governing the dry contact has been defined as follows: find $p_{\mathrm{d}}$ and $h-h_{0}$ such that

$$
\begin{aligned}
& C p_{\mathrm{d}}+\left(h_{\mathrm{g}}+h_{\mathrm{e}}-h_{0}\right)=h-h_{0} \\
& p_{\mathrm{d}} \geq 0 \\
& h-h_{0} \geq 0 \\
& p_{\mathrm{d}} \cdot\left(h-h_{0}\right)=0
\end{aligned}
$$

The formulation presented above has been then added to the EHL complementarity formulation in the presence of cavitation in order to solve the mixed lubrication problem.

In particular, the correct detection of the dry contact in an EHL lubricated bearing can be achived in two steps:

1. The complementarity EHL Reynolds equation is solved;

2. The complementarity dry contact system is solved.

The solution of the EHL Reynolds equation provides the initial gap that is used to solve the dry contact problem. In fact, when the fluid film hydrodynamic pressure is evaluated, the film thickness, $h$, calculated with Eq.5, may become negative (lower than the surface roughness) in some points of the domain, especially in the presence of very high loads. In this case the second step is performed: the total film thickness, $h$, is used to evaluate the initial gap $h-h_{0}$ and the system of linear equations in Eq.10 is solved. The resulting pressure vector, $p_{\mathrm{d}}$, presents non-null values where dry contact occurs. As a consequence, a total pressure $p_{\text {tot, }}$ is defined as the sum between the hydrodynamic pressure $p$ and the dry contact pressure $p_{\mathrm{d}}$.

\section{APPLICATION}

In this section, the EHD analysis of the small-end of the conrod of a high performance motorbike engine is presented, using the proposed mass conserving complementarity algorithm. The analysis of the small-end bearing is characterized by a prevailing squeeze motion of the lubricant while a sort of uncertainty regarding the relative sliding speed between the conrod small-end assembly components exists. The relative speed between the piston and the conrod is a function of the length of the conrod itself, of the radius of the crankshaft and of the rotational speed of the engine. It also varies during time with a sinusoidal law and different instants exist where this speed is null. Moreover, the piston and the conrod are connected by the piston pin, which usually floats with respect to the two other components. For this reason, it is not possible to define an exact relative speed between the two mating surfaces of the conrod small-end and of the piston pin. Therefore, two opposite cases are here considered: in the first case it is assumed that the piston pin is wedged into the piston, that means that there is sliding only between the piston pin and the conrod, while in the second case the pin is considered to not rotate with respect to the conrod.

\section{GOVERNING EQUATION}

Constant film thickness calculations are unsuitable for bearing analyses and more flexible and realistic implementations based on the equilibrium equations are conventionally employed. In fact, it is usually the loading history of the bearing to be known and important outputs of the analysis are the journal path, the pressure distribution and the minimum film thickness value.

For these reasons, a more sophisticated numerical algorithm, that solves simultaneously the equilibrium and the EHL Reynolds equations, has been implemented. For each time step, the pressure and void fraction fields, namely $p$ and $r$, (and possibly the dry contact pressure $p_{\mathrm{d}}$ ) are obtained by solving the Reynolds equation and they are evaluated in all the nodes of the domain. The correct position of the piston-pin centre is computed so that the resultant of the pressure distribution balances the external load.

It is possible to consider the conrod fixed and the loads applied to the piston pin (the following discussion and equations are valid also in the case of a fixed pin, where the loads are applied to the conrod). In this case, the equilibrium equations in cylindrical coordinates are:

$$
\begin{aligned}
& \int_{\varphi} p_{t o t}(\gamma, z) \cos (\gamma) d \varphi=F_{x}(t) \\
& \int_{\varphi} p_{t o t}(\gamma, z) \sin (\gamma) d \varphi=F_{y}(t)
\end{aligned}
$$

The calculation of the forces acting on the bearing is based on the geometrical properties, the mass properties and working conditions of the conrod assembly. The schematic depicted in Fig.2 shows the assembly coordinate system and its most significant dimensions.

\section{OIL SUPPLY HOLE}

The conrod small-end presents two holes to supply lubricant into the contact area, located at $\pm 45^{\circ}$ from the longitudinal axis of the conrod. Corresponding to the positions of the supply holes the value of the pressure is set equal to the oil supply pressure. 


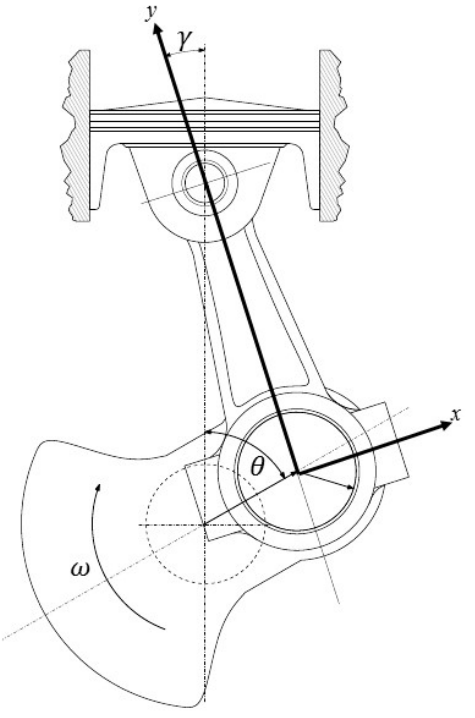

Fig.2 Schematic of the conrod assembly and of the coordinate system.

\section{OIL MODEL}

For the sake of simplicity the lubricant has been considered as a Newtonian fluid and also the piezoviscous behavior is neglected. Moreover, isothermal simulations have been carried out. The oil properties have been considered to not vary during the simulation time and they refer to the chosen working temperature. In order to investigate the influence of the lubricant properties on the behavior of the system two different oil temperature have been taken into account, namely $140{ }^{\circ} \mathrm{C}$ and $160{ }^{\circ} \mathrm{C}$. The oil properties are shown in Table 1 .

\begin{tabular}{|c|c|c|}
\hline $\begin{array}{c}\text { Temperature } \\
\left({ }^{\circ} \mathrm{C}\right)\end{array}$ & $\begin{array}{c}\text { Density } \rho \\
\left(\mathrm{kg} / \mathrm{m}^{3}\right)\end{array}$ & $\begin{array}{c}\text { Dynamic viscosity } \mu \\
(\mathrm{cP})\end{array}$ \\
\hline 140 & 777.0 & 6.3 \\
\hline 160 & 765.0 & 4.6 \\
\hline
\end{tabular}

Tab.1 Lubricant properties as a function of the temperature.

\section{ELASTIC DEFLECTION}

In Table 2 the main material properties of the assembly components are summarized.

\begin{tabular}{|c|c|c|}
\hline Component & $\begin{array}{c}\text { Young modulus } E \\
(\mathrm{MPa})\end{array}$ & Poisson ratio $v$ \\
\hline Conrod & 210.000 & 0.3 \\
\hline Piston pin & 210.000 & 0.3 \\
\hline Piston & 75.000 & 0.33 \\
\hline
\end{tabular}

Tab.2 Material properties of the assembly components.

The compliance matrix of the structure in the radial direction has been extracted from a Finite Element model of the connecting rod and the piston-pin assembly, see Fig.3. Due to the symmetry of the problem with respect to the conrod moving plane, only a half of the components has been discretized. The mesh of the Finite Element structural model has been converted and reduced via a downsampling procedure to match the EHL domain employed for the lubrication analysis. In particular, a fluid film mesh of 100 elements in the circumferential direction and 12 elements in the axial direction has been employed. Four-nodes bilinear elements have been adopted in all the simulations. Fig.4 shows the effects of a uniform unit pressure applied to all the nodes of the domain in terms of radial displacements.
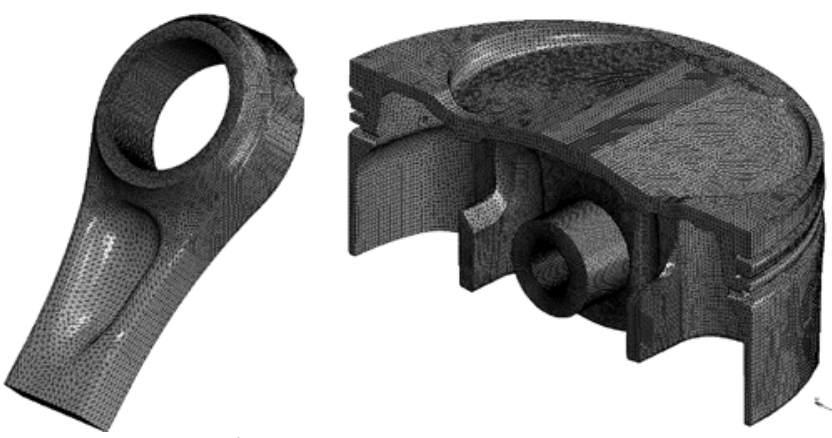

Fig.3 Finite Element discretization.

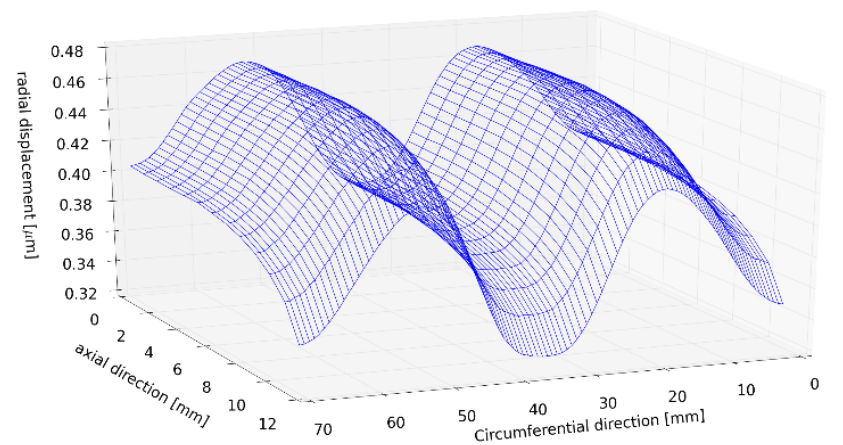

Fig.4 Radial displacement of the contact surface under the action of a uniform unit pressure applied to all the nodes of the domain.

\section{NUMERICAL ALGORITHM}

The solution of the sets of Eq.5 and 10 gives the journal centre position, $e$, at each time step of the simulation.

In order to numerically solve the EHL problem, two nested iterative loops have been implemented: the inner loop solves the EHL Reynolds equation in the pressure and void fraction variables, the journal position given, while the outer loop finds the correct journal position to match the external load. This procedure is executed for every time step. Fig.5 reports the pseudocode of the procedure.

The convergence of the solution of the inner loop is facilitated by the Newton method employed to solve the EHL Reynolds equation.

The procedure of the outer loop also adopts a Newton method, which allows a quick convergence and saves computational time. In fact, the benefits of the quadratic convergence of the Newton method overcome the cost of the evaluation of the related Jacobian matrix. 


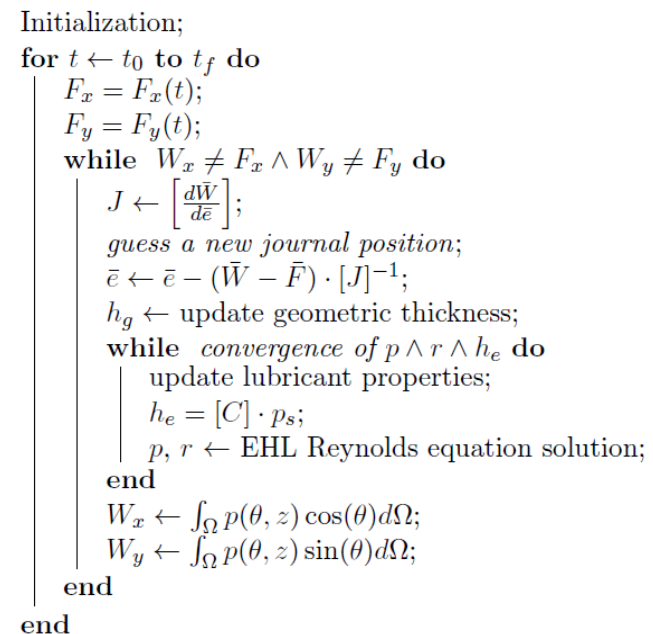

Fig.5 Pseudocode of the solving procedure.

\section{EXTERNAL LOAD}

The high rotational speed of the engine produces high inertial forces on the piston-pin assembly, while the forces due to the pressure of gases in the combustion chamber become relevant only during the compression and the power stroke. According to the adopted coordinate system, integral with the conrod, both piston-pin inertial forces and combustion forces are directed along the $y$ axis, while only the inertial forces due to the rotational motion of the conrod are directed along the $x$ axis. Fig.6 shows the loads applied to the system.
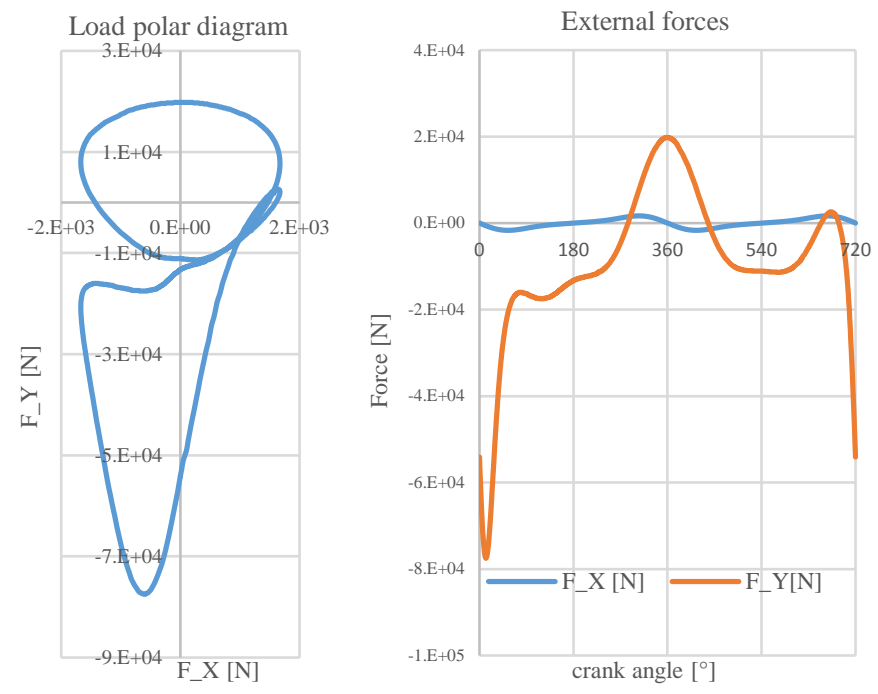

Fig.6 External forces.

\section{BEARING DATA AND BOUNDARY CONDITIONS}

Table 3 collects the main geometrical parameters of the small-end bearing investigated and the analysis conditions. All the simulations have been performed considering the engine at max power regime.

\begin{tabular}{|l|c|r|l|}
\hline Piston-pin external radius & $R_{e s}$ & 11.0 & $\mathrm{~mm}$ \\
\hline $\begin{array}{l}\text { Piston-pin internal radius } \\
\text { (reference) }\end{array}$ & $R_{i}$ & 5.5 & $\mathrm{~mm}$ \\
\hline Piston-pin internal radius (stiffer) & $R_{i s}$ & 5.0 & $\mathrm{~mm}$ \\
\hline Small-end axial length & $T$ & 11.15 & $\mathrm{~mm}$ \\
\hline Radial clearance (reference) & $c_{r}$ & 0.0175 & $\mathrm{~mm}$ \\
\hline Radial clearance (maximum) & $c_{M}$ & 0.026 & $\mathrm{~mm}$ \\
\hline Radial clearance (minimum) & $c_{m}$ & 0.01 & $\mathrm{~mm}$ \\
\hline Roughness (reference) & $r o$ & 0.7 & $\mu \mathrm{m}$ \\
\hline Roughness (smoother) & $r o s$ & 0.25 & $\mu \mathrm{m}$ \\
\hline Crankcase pressure & $p_{a m b}$ & 0.05 & $\mathrm{MPa}$ \\
\hline Cavitation pressure & $p_{c}$ & 0.0 & $\mathrm{MPa}$ \\
\hline Supply oil pressure & $p_{\text {sup }}$ & 0.05 & $\mathrm{MPa}$ \\
\hline Rotational speed & $r p m$ & 10500 & \\
\hline
\end{tabular}

Tab.3 Main simulation parameters.

\section{RESULTS}

This section describes the results obtained.

\section{Radial clearance}

First, a comparison between three cases with different radial clearance values is reported. Fig.7 depicts the orbit diagrams for the three cases analyzed.

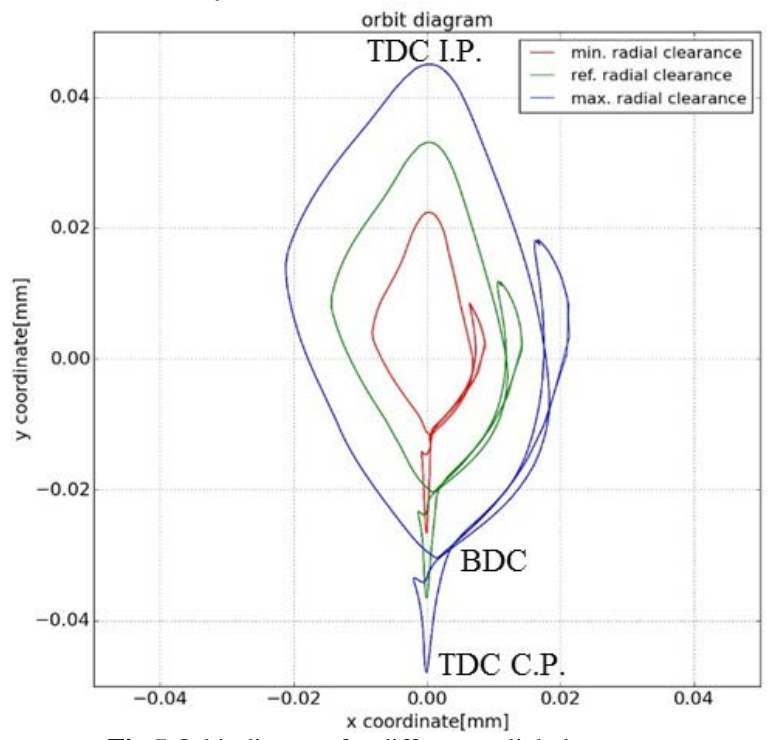

Fig.7 Orbit diagram for different radial clearances.

As expected the motion of the centre of the piston-pin is strongly affected by the radial clearance value. Fig.8 shows the minimum film thickness registered in the three cases. For each case three curves are displayed: the first curve refers to the minimum film thickness registered along the nodes on the conrod symmetry plane, namely sym, the second refers to a row of nodes along a 
mid-plane between the symmetry plane and the external plane of the conrod, namely mid, and the last curve refers to a row of nodes on the external side of the conrod, namely ext.
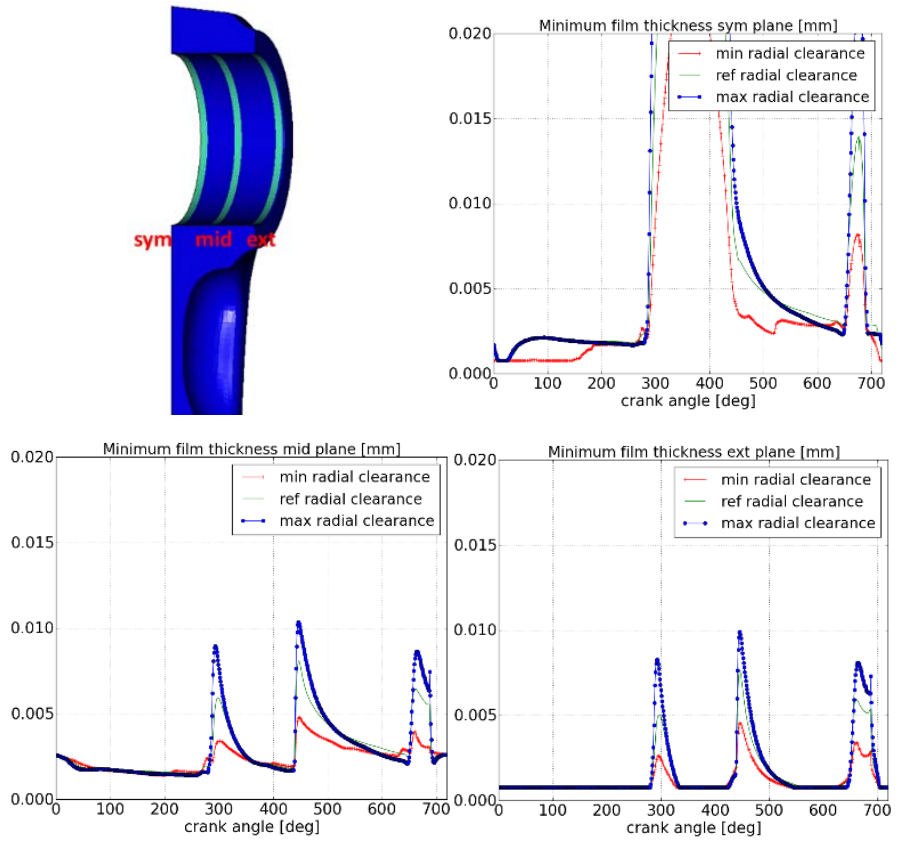

Fig.8 Minimum film thickness for different radial clearances.

When the value of the minimum film thickness equals the roughness value it means that in some nodes of the domain dry contact occurs. It is clear that a smaller value of the radial clearance produces a higher load carrying capacity and as a consequence the intensity of dry contact pressure is reduced. Fig.9 reports the distribution of the mean value of the total pressure during the engine cycle for the three cases analyzed.

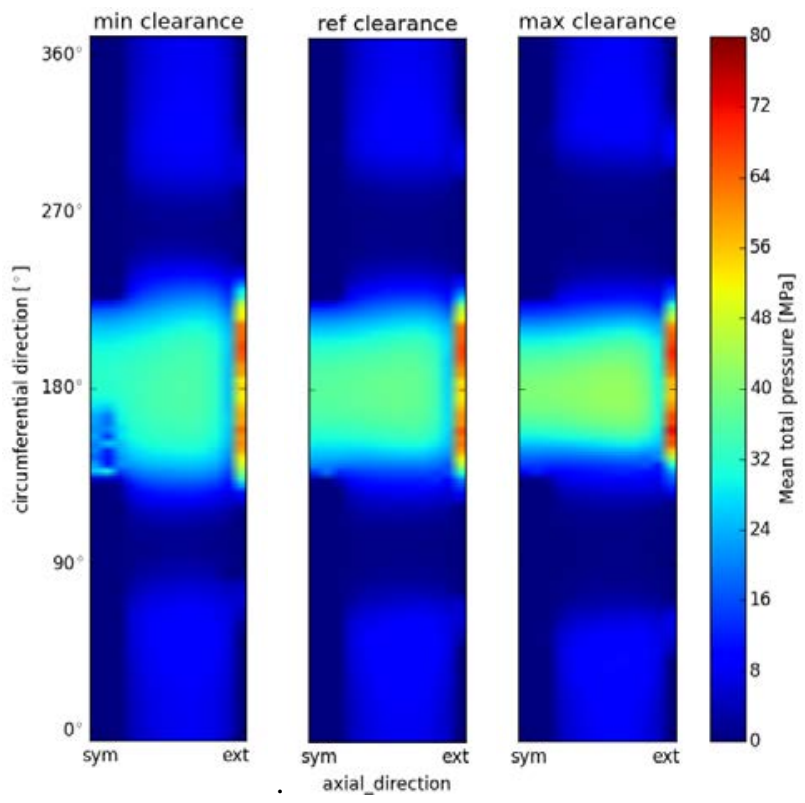

Fig.9 Mean total pressure for different radial clearances.
With an increasing of the radial clearance a higher localization of the total pressure occurs. Moreover in the presence of a larger clearance, the amount of dry contact increases and quicker wear phenomena can arise.

\section{Lubricant temperature}

A second aspect investigated is the influence of the lubricant temperature on the bearing tribological behavior. Two different oil temperature $\left(140^{\circ} \mathrm{C}\right.$ as reference temperature, and $\left.160^{\circ} \mathrm{C}\right)$ have been simulated. The operating temperature affects the oil viscosity value and, as a consequence, the load capacity of the bearing. In Fig.10 the comparison between the two cases simulated is reported in terms of orbit diagram.

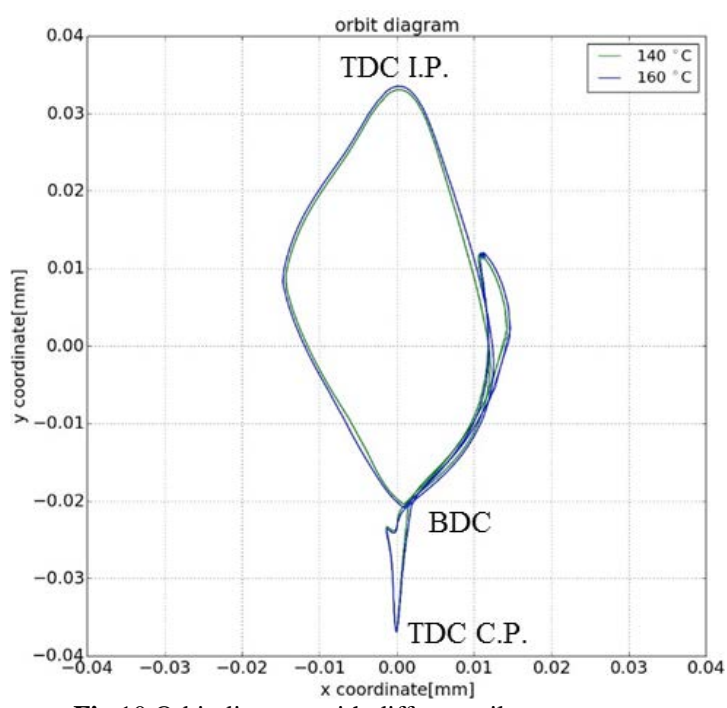

Fig.10 Orbit diagram with different oil temperatures.

A very little variation have been observed. Fig.11 shows a comparison in terms of the distribution of the mean total pressure during the entire engine cycle.

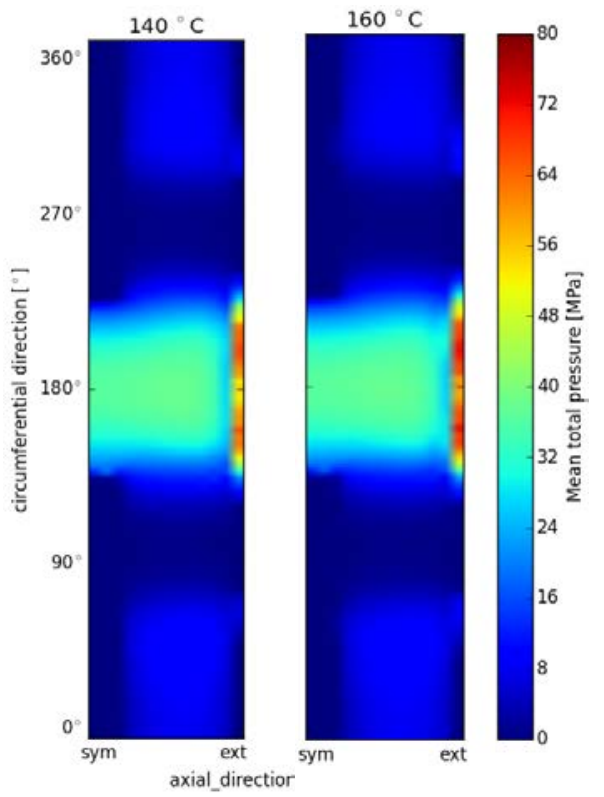

Fig.11 Mean total pressure with different lubricant temperatures. 
Increasing the lubricant temperature, the contact pressure on the external side of the bearing reaches higher values and, as a consequence, the reliability of the component can be compromised.

\section{Surface roughness}

A third parameter considered is the roughness of the mating surfaces. In fact, lower roughness values allow to sustain higher external loads avoiding dry contact pressure. To quantify the influence of this parameter, two different values of roughness ( $r o=0.7 \mu \mathrm{m}$ and $r \mathrm{~s}_{\mathrm{s}}=0.2 \mu \mathrm{m}$ ) have been simulated. In particular, $r o$ represents the standard roughness value of the coupling surfaces, while $\mathrm{ro}_{\mathrm{s}}$ is a smoother value that could be obtained with a finer (and more expensive) surface finishing. Fig.12 shows a comparison in terms of mean dry contact pressure distribution during the engine cycle.

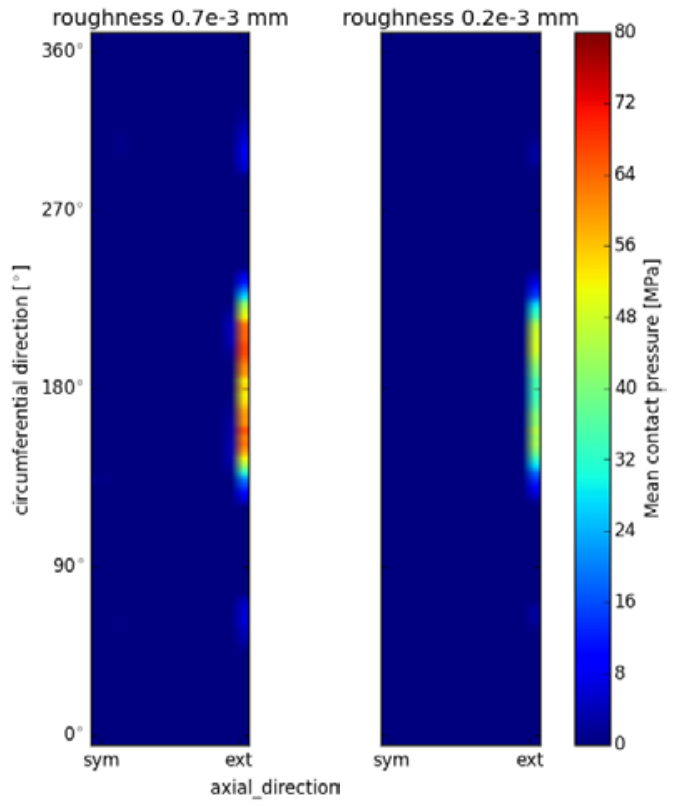

Fig.12 Mean dry contact pressure with different surface roughness.

It is evident that in the case with roughness $r o_{\mathrm{s}}$ the maximum value of the mean contact pressure is lower than in the case with the roughness ro.

\section{Piston-pin stiffness}

To improve the assembly tribological performance without modifying its manufacturing process to reduce the surface roughness, a different pin has been designed. In particular, the new pin has the same external radius of the previous one but presents a $1 \mathrm{~mm}$ lower internal diameter, thus increasing the stiffness of the component.

Making the piston-pin even stiffer, thus further decreasing the elastic deflection of the component, the tribological behaviour of the assembly could be further improved. Nevertheless, an increase of the mass of the component produces a consequent augment of the inertial forces. In engines operating at very high rotational speed like the one under investigation in this work, the entity of the inertial forces is a very important aspect to be taken into account. In particular, to limit the mass of alternating components is a crucial point.

The modification of the inner radius of the piston pin has a strong influence on the assembly performance. In Fig.13 the comparison in terms of the orbit diagram is reported.

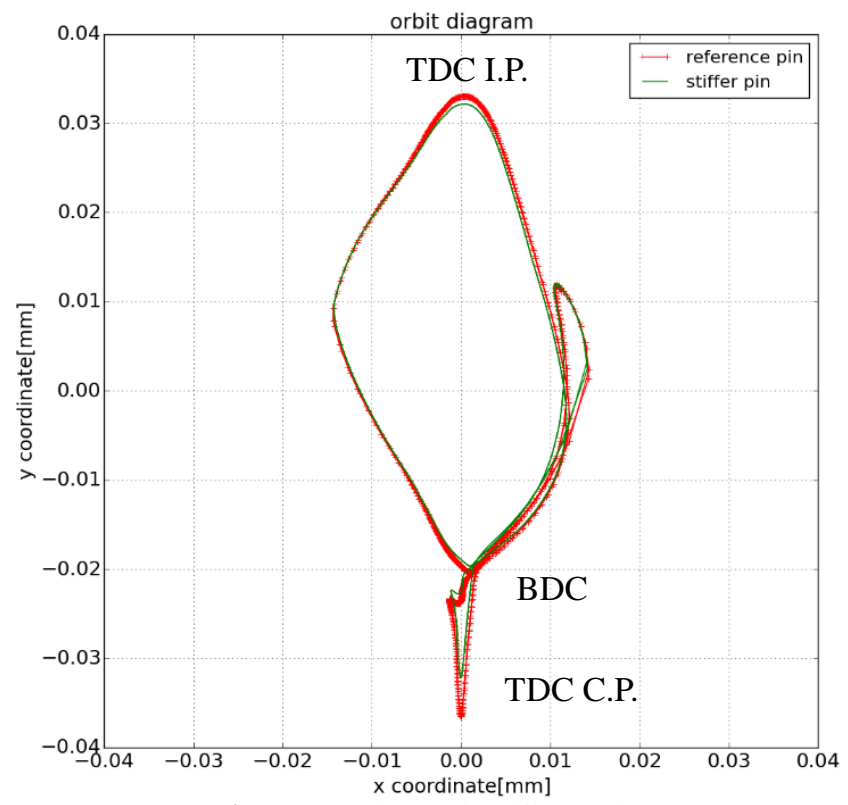

Fig.13 Orbit diagram for different pins.

The main differences are registered during the combustion phase and when the piston is near the TDC at the beginning of the induction stroke. In both cases, the displacement of the pin centre is lower when the stiffer pin is considered, because of the minor elastic deflection of the component.
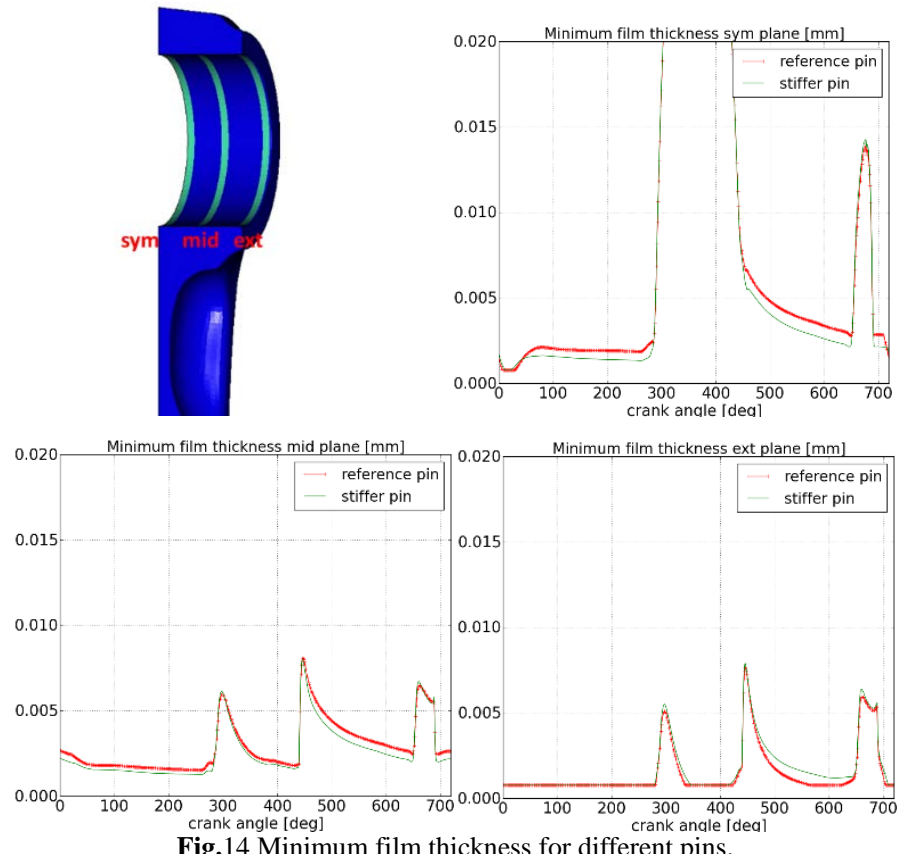

Fig.14 Minimum film thickness for different pins. 
Fig.14 shows a comparison in terms of the minimum film thickness distribution during the engine cycle. It is interesting to note how the stiffer pin produces a lower value of the minimum film thickness in almost all the domain except for the external plane where the dry contact occurs. This result is highlighted also by the mean total pressure distribution, see Fig.15.

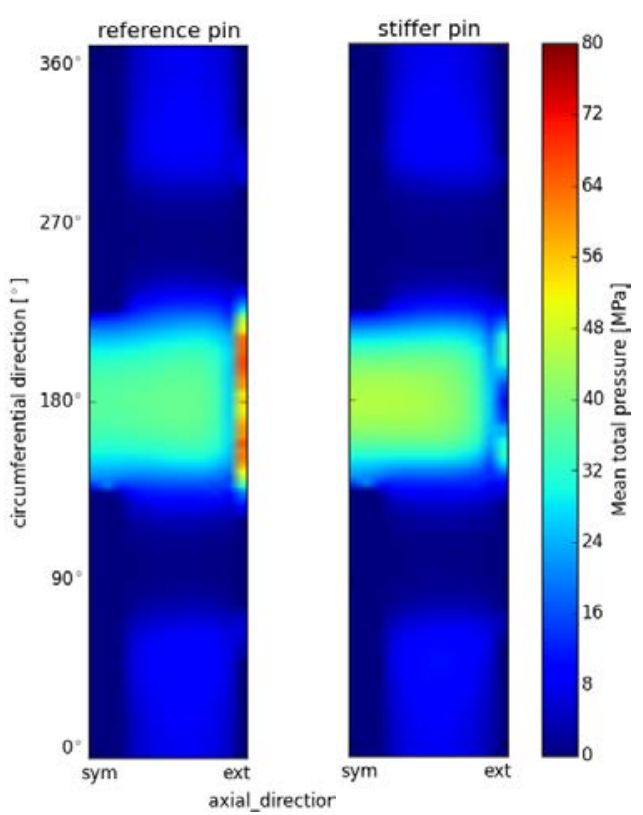

Fig.15 Mean total pressure for different pins.

The new pin configuration provides a better pressure distribution and it allows to strongly reduce the amount of mean dry contact pressure on the external plane of the bearing.

\section{Relative sliding speed}

Looking at the diagrams of Fig.6, the magnitude of the transverse forces acting on the conrod small-end bearing is almost negligible with respect the longitudinal forces. This condition could suggest to build a two symmetrical model exploiting another plane of symmetry of the assembly, orthogonal to the motion plane of the conrod. Nevertheless, to actually adopt this model, it is important to verify that also the relative sliding speed between the pin and the conrod only marginally affects the tribological behavior of the system. In this prospective a simulation has been carried out neglecting the sliding speed between the mating surfaces. No relevant changes have been encountered both in terms of pin centre path and of pressure distribution, see Fig.16 and Fig.17.

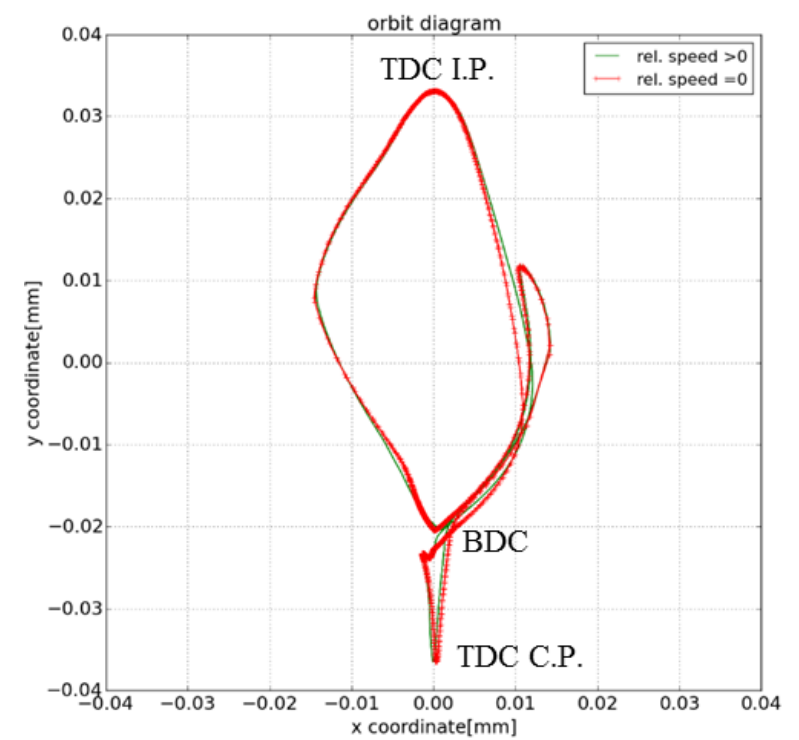

Fig.16 Orbit diagram for the case with relative sliding speed and for the case without relative sliding speed.

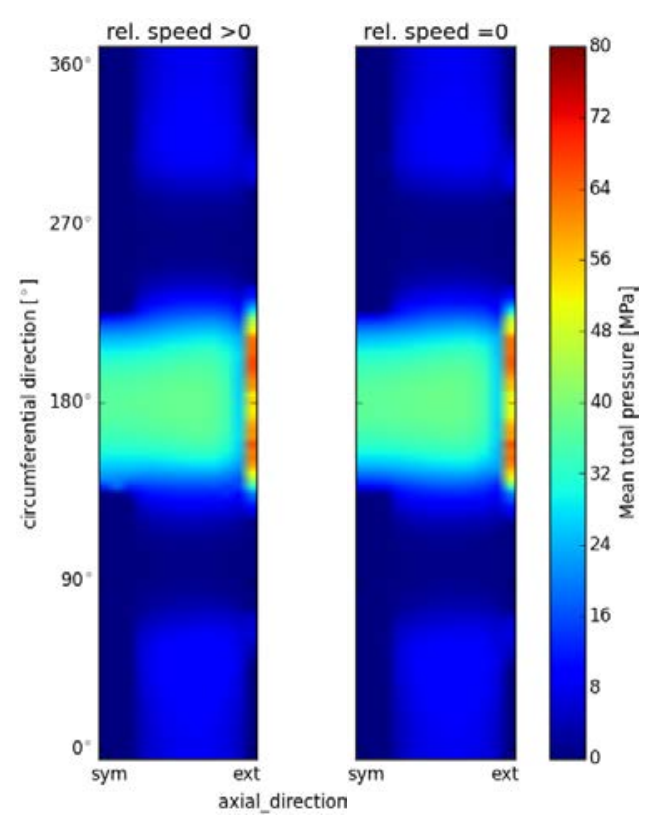

Fig.17 Mean total pressure distribution for the case with relative sliding speed and for the case without relative sliding speed.

Moreover, the results of Fig.17 suggest that the pressure distribution is almost symmetrical with respect to the plane orthogonal to the motion plane of the conrod thus corroborating the idea that both the transversal forces and the relative sliding speed do not have relevant effect on the bearing behaviour.

\section{Two symmetrical model}

Therefore, a two symmetrical model has been prepared. 

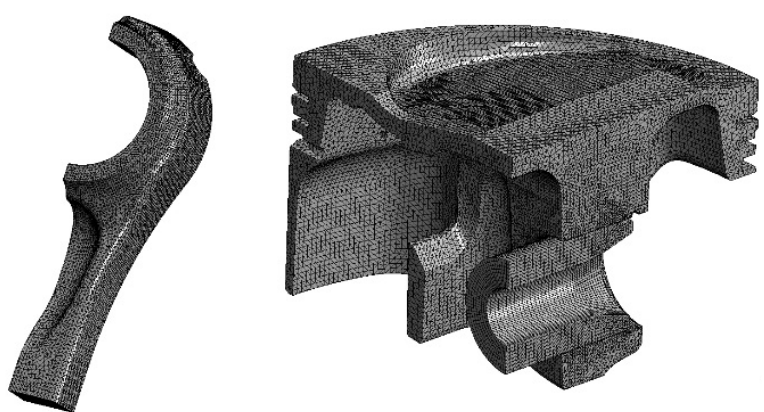

Fig.18 Finite Element discretization of the two symmetrical model.

The model presents a fluid film mesh of 50 elements in the circumferential direction (a half of the simply symmetrical model) and 12 elements in the axial direction (the same of the simply symmetrical model). The reduction of the number of nodes allows a strong reduction in the computational time (ten times lower). The results obtained show good correlation with the simply symmetrical model both in terms of minimum film thickness and of pressure distribution. It means that this approach could be very useful in the design phase of the component to evaluate the effect of the geometrical parameters of the assembly or of a variation of external loads.

Fig.19 reports the comparison between the simply symmetrical model and the two symmetrical model in terms of the pin centre path.

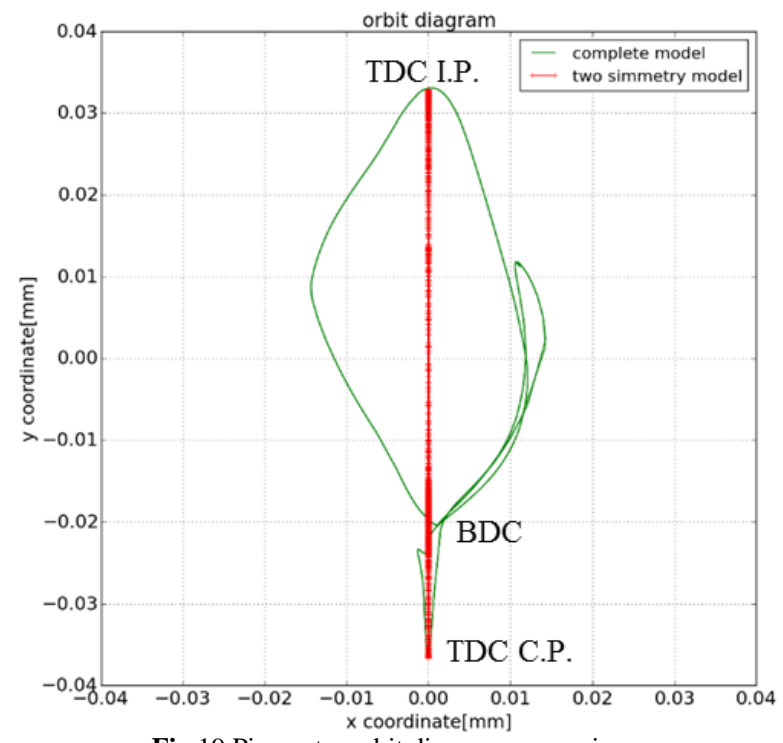

Fig.19 Pin centre orbit diagram comparison.

The orbit diagram of the two symmetrical model presents the same maximum displacement of the centre of the pin registered for the simply symmetrical model during the combustion phase and at the TDC at the beginning of the induction stroke. Due to the additional symmetry constrain, the pin cannot move in the transverse direction. In this case, the pin describes a pure squeeze motion.

Also in terms of pressure distribution a good agreement has been reached, see Fig.20.

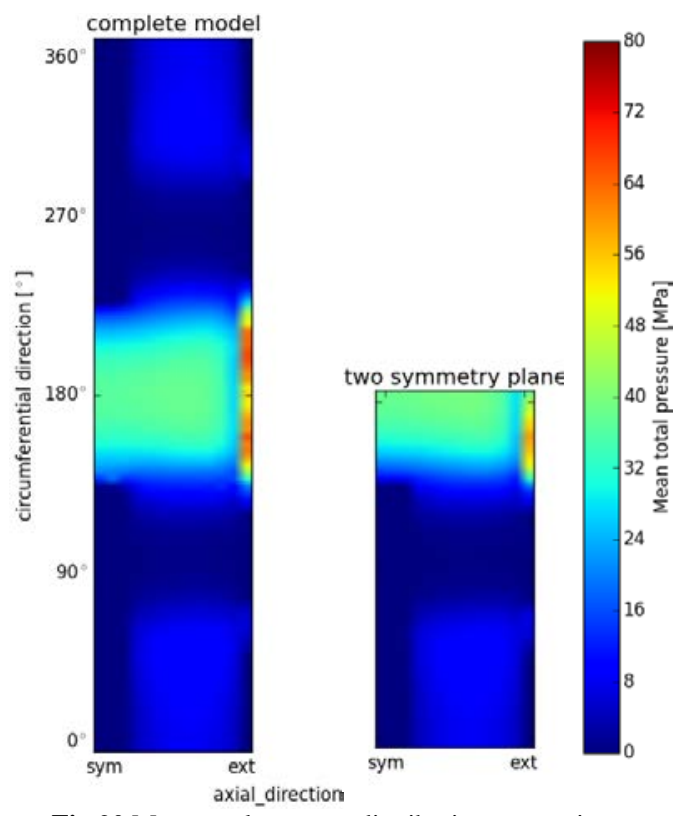

Fig.20 Mean total pressure distribution comparison.

To better appreciate the agreement between the results obtained by the two models, a 3D plot of the total pressure at TDC during the combustion phase and at TDC at the beginning of the induction stroke are reported in Fig.21.

TDC combustion phase

Simply symmetrical model

Two symmetrical model
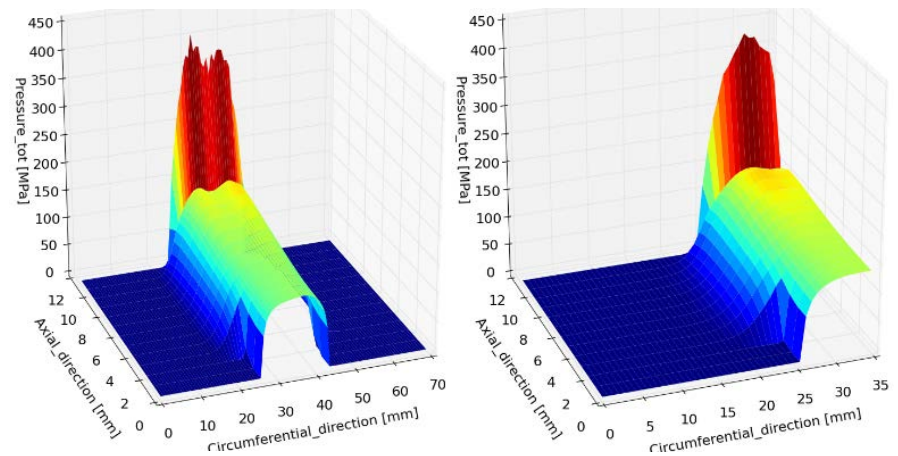

TDC induction phase

Simply symmetrical model

Two symmetrical model
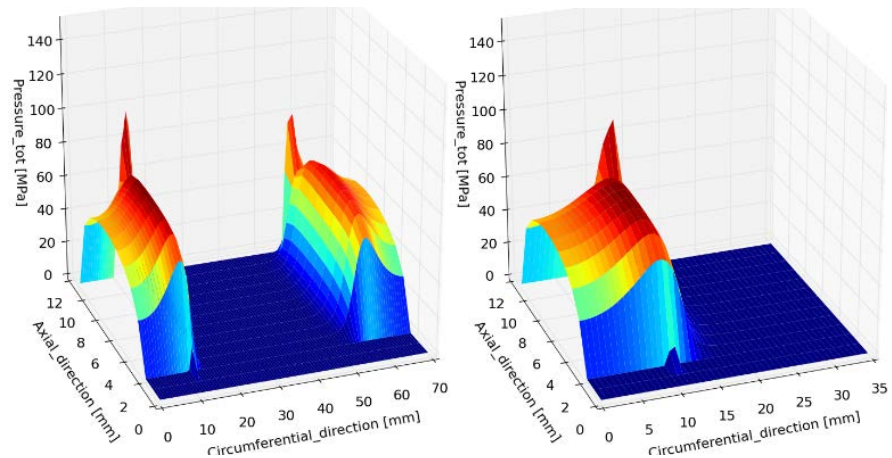

Fig.21 Total pressure distribution comparison. 


\section{CONCLUSIONS}

In this contribution a complementarity formulation for the solution of EHL problem in presence of cavitation has been employed in order to investigate the tribological behavior of the conrod small-end of a high performance motorbike engine. The influence of different physical and geometrical parameters have been discussed. In particular, the clearance between the conrod small-end and the piston pin, the lubricant physical properties, the surface roughness and the stiffness of the piston pin have been investigated, thus providing preliminary guidelines for the correct design of the coupling. Due to the negligible influence of the transversal forces and of the relative sliding speed between the mating surfaces, a two symmetrical model of the assembly has been prepared and results have been compared with those obtained adopting a simply symmetrical model. A good agreement has been observed between the results favoured by the two models.

\section{REFERENCES}

[1] Marmorini, L., Baldini, A., Bertocchi, E., Giacopini, M., Rosi, R., Strozzi, A. "On the loosening mechanism of a bush press-fitted in the small end of a connecting rod", $P$. I. Mech. Eng. D-J AUT. 03/2012, 226(3):312-324.

[2] Aitken, M. B. and McCallion, H. "Elastohydrodinamic Lubrication of Big-End Bearings, Part 1: Theory”, Proc. IMechE, Part C: J. Mechanical Engineering Science, 1991, 205, 99-106. DOI: 10.1243/PIME_PROC_1991_205_097_02.

[3] Fantino, B., Frêne, J. and du Parquet, J., "Elastic connecting-rod bearing with a piezoviscous lubricant: analysis of the steady-state characteristics”, Trans. ASME, J. Lubrication Technol., 101, 1979, 190-200.

[4] Giacopini, M., Bertocchi, L., Baldini, A., Dini, D., “A Complementarity Formulation for the EHL Analysis of a Conecting Rod Big End Bearing”, 2013, 5 ${ }^{\text {th }}$ WTC, September 8-13, Torino, Italy.

[5] Dini, D., Mastrandrea, L. N., Giacopini, M., Bertocchi, E., "Numerical Investigation of the Cavitation Damage in a High Performance ENGINE Conrod Big End Bearing Via a Mass-Conserving Complementarity Algorithm”, 2014, STLE Annual Meeting \& Exhibition, May 18-21, Lake Buena Vista, Florida, USA.

[6] Wang, D., Mian, O., Merritt, D., Zhu, G., “ElastoHydrodinamic Lubrication Analysis and Wear Predction of Connecting Rod Small-End Bush Piston Pin Interface”. SAE Technical Paper 2008-36-0068, 2008, doi: 10.4271/2008-36-0068.

[7] Ligier, J. and Ragot, P., "Small End Conrod Lubrication" SAE Technical Paper 2006-01-1101, 2006, doi: 10.4271/2006-01-1101.

[8] Spuria, M., Bonneau, D., Le Baratoux, Y., Molari, P.G., "A dynamic Model for an Internal Combustion Engine Full-floating Piston Pin in Lubricated Conditions”, XVII Congresso dell'Associazione Italiana di Meccanica Teorica e Applicata, Firenze 11-15 Settembre 2015.
[9] Bonneau, D., Optasanu, V., "Finite Element MassConserving Cavitation Algorithm in Pure Squeeze Motion. Validation/Application to a Connecting-Rod Small End Bearing”, Journal of Tribology, January 2000, Vol. 122, pp. 162-169.

[10] Bertocchi, L., Giacopini, M., Dini, D., “Analisis of the Lubrication Regimes at the Small End and Big End of a Connecting Rod of A High Performance Motorbike Engine”, Proceedings of the ASME/STLE 2012 International Joint Tribology Conference, October 7-10, 2012, Denver, Colorado, USA.

[11] Boedo, S., Booker, J. F., "Cavitation in normal separation of square and circular plates”, J. Tribol. 117 (1995), pp. 403-410.

[12] Bertocchi, L., Giacopini, M., Dini, D., Fowell, M., Strozzi, A., “A Mass-Conserving Complementarity Formulation to Study Fluid Film Lubrication in the Presence of Cavitation for Non-Newtonian and Compressible Fluids”, J. Tribol.-T. ASME, 132(4), pp. 041702.

[13] Giacopini, M., Dini, D., Fowell, M., Strozzi, A., “A MassConserving Complementarity Formulation to Study Lubricant Films in the Presence of Cavitation”, J. Tribol.T. ASME, October 2010, Vol. 132/041702-1.

[14] Ruskell, L. E. C., "A rapidly converging theoetical solution of the elastohydrodynamic problem for recta3ngular rubber seals" Journal of Mechanical Engineering Science, 22, 1, 1980, 9-16.

[15] Bertocchi, L., “A Linear Complementarity Approach to Solve Elastohydrodinamic Lubrication Problems in the Presence of Cavitation”, PhD Thesis, March 2013.

[16] Strozzi, A., 1985, "Formulation of Three Lubrication Problem in Terms of Complementarity”, Wear, 140(2), pp. 103-119. 